\title{
Coping with the metabolic stress of leaving home
}

\author{
Cell Research (2016) 26:757-758. doi:10.1038/cr.2016.61; published online 24 May 2016
}

\begin{abstract}
Detachment from extracellular matrix causes metabolic defects that transformed cells must overcome in order to survive and proliferate outside of their normal niche. A recent report from Jiang et al. published in Nature describes how cancer cells grown in suspension utilize reductive carboxylation of glutamine to transfer reducing power from the cytosol to mitochondria to detoxify reactive oxygen species and promote anchorage-independent growth and survival.
\end{abstract}

In organized tissues, epithelial cell functions are controlled by extracellular cues, including those transmitted through physical contact with the extracellular matrix (ECM). Attachment to ECM dictates the structural organization of tissues and promotes epithelial cell survival and proliferation. When normal cells lose contact with ECM, they undergo a form of apoptotic cell death known as anoikis - this mechanism of tissue homeostasis ensures that epithelial cells only grow when and where they will maximally benefit the organism as a whole. During the early stages of oncogenic transformation, aberrant proliferation of newly transformed cells physically displaces them from the ECM, forcing selection of cells that have developed mechanisms to allow survival and proliferation outside of their normal niche. The ability to avoid anoikis - that is, to become anchorage independent - is an important characteristic of most epithelial tumors that is also important at later stages, when metastasizing cancer cells leave their tissue of origin and colonize distant sites with unique ECM environments that typically would not support their survival.

An essential component of anchorage independence is the ability to overcome the metabolic defects associated with the loss of ECM attachment. When normal cells lose contact with ECM, nutrient uptake is reduced and metabolic pathways are rewired, leading to decreased biosynthesis and compromised bioenergetics $[1,2]$. This metabolic remodeling is due, at least in part, to decreased PI3K/ AKT and MEK/ERK signaling, which is normally facilitated by ECM attachment. Expression of oncogenes that maintain signaling upon matrix detachment can partially rescue these metabolic defects, a function that is required to promote survival in the absence of ECM contact [1].

An interesting aspect of the metabolic changes associated with loss of matrix attachment is a strong induction of reactive oxygen species (ROS) [1]. ROS, such as superoxide and hydrogen peroxide, are byproducts of normal cellular processes and are detoxified by cellular antioxidants like the thioredoxin and glutathione systems. In turn, these antioxidants can be regenerated using the reducing power from the metabolite $\mathrm{NADPH}$, which is generated by multiple metabolic pathways throughout the cell. While the role of ROS in cancer is complex, several recent reports have clearly shown that cellular antioxidant systems are required for multiple steps of tumorigenesis, including tumor initiation [3] and metastasis [4], both of which require anchorage-independent growth. This suggests that the ability to detoxify ROS upon ECM detachment is a critical component of anchorage independence and essential for tumor development and progression. Indeed, provision of exogenous antioxidants can promote anoikis resistance [1], metastasis [4], and cancer incidence [5].

To investigate metabolic differences between tumor cells growing in the presence and absence of ECM, Jiang et al. [6] compared the metabolism of cancer cells grown in traditional 2D culture to those grown in suspension as spheroids. Among the most significant differences was a large increase in the reductive carboxylation of glutamine in cells grown as spheroids. Reductive glutamine metabolism was recently shown to play an important role in providing citrate and acetyl-CoA for lipid synthesis during hypoxia or when mitochondrial metabolism is otherwise impaired [7-9]. Interestingly, citrate generated by reductive glutamine metabolism in spheroids was not utilized for lipid synthesis; rather, reductive carboxylation was important for mitigating mitochondrial ROS. This finding was unexpected given that reductive carboxylation of glutamine to generate citrate requires the consumption of NADPH, which would be predicted to exacerbate ROS rather than help to detoxify it. Jiang et al. began to address this dichotomy by demonstrating that knockout of the cytosolic IDH1 was sufficient to block reductive metabolism in spheroids, while loss of mitochondrial IDH2 had a minimal effect. It has been suggested that mitochondrial IDH2-mediated reductive carboxylation could serve as part of an NADPH shuttle where citrate in the mitochondria is transported to the cytosol to be oxidized by IDH1 to generate NADPH [10]. Jiang et al. provided evidence that this shuttle runs in reverse in spheroids, mediating the transfer of cytosolic NADPH into the mitochondria (Figure 1). This conclusion was supported by the evidence that while 


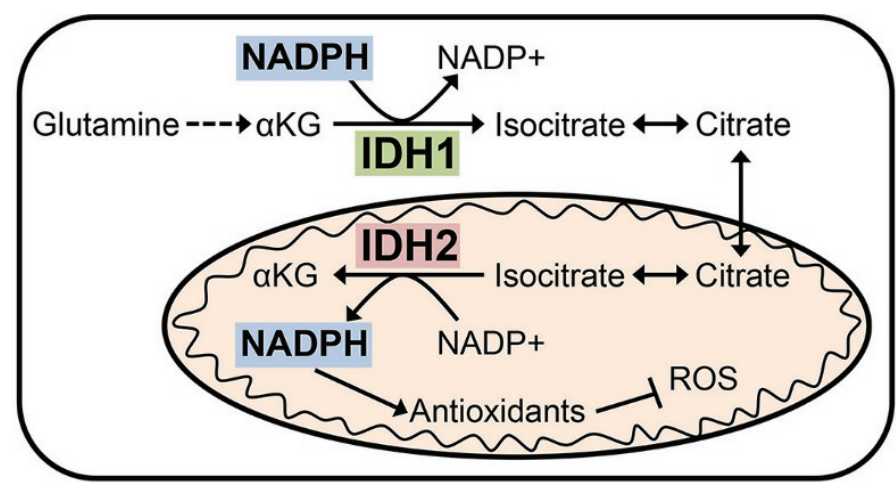

Figure 1 Reductive carboxylation of glutamine mediated by IDH1 facilitates the transfer of reducing power in the form of NADPH from the cytosol to mitochondria to help detoxify ROS generated in tumor cells grown in spheroids.

knockout of IDH2 had a minimal effect on reductive carboxylation, it was still required for abrogation of mitochondrial ROS.

NADPH provides reducing power not only for antioxidant systems, but also for many biosynthetic reactions, and has even been postulated to be the limiting metabolite for cell proliferation. Given the importance of these processes in cancer cells, there has been considerable interest in understanding how cancer cells generate NADPH. Studies addressing this question have identified key roles for multiple metabolic pathways that contribute to cellular NADPH pools, including the pentose phosphate pathway (PPP) and the folate pathway $[11,12]$. Key to these studies has been the use of deuterium $\left({ }^{2} \mathrm{H}\right)$ tracers that allow quantitative analysis of NADPH biosynthesis. A complicating factor, however, is the existence of distinct cytosolic and mitochondrial pools of NADPH that are difficult to distinguish experimentally. One approach that has been employed takes advantage of the neomorphic activity of mutant IDH1/2 that generates the oncometabolite 2-hydroxyglutate (2-HG). ${ }^{2} \mathrm{H}$ from NADPH is transferred to 2-HG by mutant IDH1 or IDH2, with the subcellular localization of these enzymes allowing the synthesis of 2-HG from distinct subcellular pools of NADPH [12]. Jiang et al. leveraged this system to demonstrate that cytosolic NADPH could be passed to the mitochondria by showing that ${ }^{2} \mathrm{H}$ from NADPH generated by the PPP in the cytosol can be detected in 2-HG made in the mitochondria by mutant IDH2, but only in cells grown in spheroids. This evidence, coupled with dependence on IDH1 and IDH2 for tumor spheroid growth that could be rescued with antioxidants, suggests that this cytosolto-mitochondria NADPH shuttle is an important mechanism for the survival and proliferation of cancer cells growing outside of their normal niche.

In addition to identifying a novel function for reductive carboxylation in combating ROS, the study by Jiang et $a l$. reinforces the importance of studying cellular metabolism in culture models that recapitulate the diverse cellular contexts that tumor cells encounter in vivo. While the tumor cells used in this study were capable of anchorage-independent growth, loss of ECM contact still substantially altered their metabolic activity, leading to increased mitochondrial ROS. Despite showing that inhibition of pyruvate dehydrogenase upon ECM detachment contributed to the induction of reductive carboxylation, it remains unclear what mechanisms are responsible for the increase in mitochondrial
ROS in suspension. This is particularly interesting given that mitochondria are a major source of both ROS and NADPH, and contain their own antioxidant systems. Further studies are needed to determine how mitochondrial ROS- and NADPH-generating pathways are altered by growth in suspension, and why they do not provide sufficient NADPH to combat ROS, leading to dependency on cytosolic reductive carboxylation. Finally, like other recent studies exploring the role of antioxidants in vivo, it is important to understand how the pathway identified by Jiang et al. contributes to different stages of tumor initiation and progression when anchorage-independent growth is predicted to be required for tumor cell survival.

\section{Jonathan L Coloff ${ }^{1}$, Joan S Brugge ${ }^{1}$}

${ }^{1}$ Department of Cell Biology, Ludwig Center at Harvard, Harvard Medical School, Boston, MA 02115, USA

Correspondence: Joan S Brugge

Tel: +1 617432 3974; Fax: +1 6174323969

E-mail: joan_brugge@hms.harvard.edu

\section{References}

1 Schafer ZT, Grassian AR, Song L, et al. Nature 2009; 461:109-113.

2 Grassian AR, Metallo CM, Coloff JL, et al. Genes Dev 2011; 25:1716-1733.

3 Harris IS, Treloar AE, Inoue S, et al. Cancer Cell 2015; 27:211-222.

4 Piskounova E, Agathocleous M, Murphy MM, et al. Nature 2015; 527:186-191.

5 Klein EA, Thompson IM Jr, Tangen, CM, et al. JAMA 2011; 306:1549-1556.

6 Jiang L, Shestov AA, Swain P, et al. Nature 2016; 532:255-258

7 Metallo CM, Gameiro PA, Bell EL, et al. $\mathrm{Na}$ ture 2012; 481:380-384.

8 Mullen AR, Wheaton WW, Jin ES, et al. Nature 2012; 481:385-388.

9 Wise DR, Ward PS, Shay JE, et al. Proc Natl Acad Sci USA 2011; 108:19611-19616.

10 Sazanov LA, Jackson JB. FEBS Lett 1994; 344:109-116.

11 Fan J, Ye J, Kamphorst JJ, et al. Nature 2014; 510:298-302.

12 Lewis CA, Parker SJ, Fiske BP, et al. $\mathrm{Mol}$ Cell 2014; 55:253-263. 